\title{
Sciendo
}

DOI: $10.2478 /$ jolace-2019-0012

\section{Test Banking in English for Medical Purposes (Dentistry)}

\author{
Ilina Doykova \\ Medical University ‘Prof. d-r Paraskev Stoyanov' in Varna, Bulgaria \\ ilina.doykova@mu-varna.bg
}

\begin{abstract}
Activities and questions to assess knowledge in English for medical purposes were designed to incorporate terminology, academic vocabulary and grammar items in computer-based tests for bachelor students in Dentistry at Medical University - Varna. During the two-semester study course identification of key words, core lexical patterns, specific collocations and emphasis on their recurrent use were the selected strategies for student retention of specialized language and improved learning outcomes. Medical terms as single-word lexical units with straightforward definitions are easy to learn compared to collocations and multi-word terms including polysemous adjectives. In the present paper we concentrate on the process of formation of structures containing attributive adjectives which can be quite a problematic and error-generating area for second language learners. The aim of the on-going research study is to extract current lists of the sub-technical vocabulary and the terminological units in specialized medical domains as linguistic resources. The collection of assessment materials into a test bank for specific educational purposes is a customizable electronic resource, imported into the University platform to facilitate the process of compilation and creation of new tests.

By implementing corpus linguistic tools into test design, the instructor aims at providing an authentic e-assessment environment based on the idea of key words in context, concordances, and lexical patterns as per the contents of the selected textbooks and teaching materials during the course. The paper highlights some strategic issues about creating test resources in EMP such as the adherence to a set of selected linguistic items and grammatical structures based on their frequency in the domain.

Key words: key words in context, lexical patterns, specialized language assessment, computer-based tests

\section{Introduction}

In the present paper we aim to reveal the potential of corpus linguistic tools in the creation of computer-based tests and to demonstrate the importance of specific lexical patterns in teaching and testing medical English at tertiary level. The specific vocabulary in the domain of dentistry - verbs, nouns, attributive and predicative adjectives that take modification and complementation - is extracted from a self-compiled medical corpus and incorporated into assessment tasks. The
\end{abstract}


key word frequency profiles are calculated automatically per concordance lines and are dependent on the patterns they participate in. The contextual use of certain lexical patterns is identified for the purpose of creating test banks for the assessment of student knowledge of specialized vocabulary. The quantitative research investigates the pairings of form and meaning that are language specific in the field of medical English (the sublanguage of Dentistry) and reveals the complexity of the valency properties of the core structures in the field at an intermediate level of English language competence (B2). The contents of the textbook in English for Dental Medicine is representative of the syllabus in terms of reading topics, development of language skills and provision of language practice. Therefore, together with the respective materials, used in the teaching process during the semesters, it is selected as a corpus for the tasks included in the final students' assessment. Two lexical research tools (WordSmith Tool, version 6 and Sketch Engine) were used to perform a word list, key word (KWIC), cluster and pattern search in the textbook contents and allow for a comparison with a reference corpus (BNC), comparison of word profiles and extraction of sample sentences.

Thus, the aims and objectives of the final assessment align with the relevant syllabus and the specificity of the corpus collection. Any test bank is self-contained (uploaded on the university learning platform Blackboard) and sufficient to address effectively the learning targets of the curriculum. Besides specific nouns and verbs, adjectives used in medical texts, were not arbitrary. These core lexical and grammatical structures of the subject area were incorporated in assessment tasks. The effectiveness of every progress or final test was indicated by analyzing the test results and performing an end-of-year survey. This approach guaranteed relevant feedback and an accurate evaluation of students' progress. The level of difficulty of the test was consistent with the curriculum requirements as consideration was given to students' accumulated knowledge and progress during the language course. The approach allowed for no recurrent use of tests, stereotyping or random choice of test content.

\section{Materials development}

In accordance with the guiding principles for quality textbooks and teaching materials, interactive questions enhance the effectiveness of the assessment. Therefore, multimedia content (appropriate video clip) was included in the test to foster students' comprehension and successful test completion. Key terms and core phraseology from the textbook were also included as per the glossary of terms and the corpus keyword list (Table 1). Additionally, students were encouraged to perform further reading tasks, and related websites were added on Blackboard (including self-study materials) to practice listening and use of English skills. 
Tab. 1: Key word extraction (Sketch Engine)

Term

suture

malocclusion

maxillary

mandibular

occlusal

caries

denture

Orthod

incisor

molar
Score Freq Ref freq

$1585.63 \quad 3920 \quad 37345$

$12521343 \quad 3349$

$1008.211189 \quad 5944$

$665.26 \quad 779 \quad 5753$

$603.09 \quad 651 \quad 3533$

$\begin{array}{lll}464.82 & 667 & 12181\end{array}$

$452.11 \quad 1518 \quad 58890$

$425.15 \quad 398$

$394.59 \quad 509$

$388.89 \quad 683$
67

8667

19997

\section{Identification of key words and lexical patterns}

In the present paper the focus was on three functional groups: verbs, nouns and especially adjectives, frequent in medical texts as they provide precision by explaining an attribute of a noun, predicate a characterizing quality or modify the subject of the sentence. Adjectives are lexical units with a collocational potential (or valency properties) where one or more positions/slots around the head word are to be filled either with obligatory, optional or contextually optional elements. In the medical corpus predicative adjectives most often occur as complements to the verbs be, become and make. Complements can be single lexical units, phrases or clauses respectively. The number of valency complements for separate lexical items varies from zero-valency (or general use with no complements) to a maximum number (mono-, di-, tri-, etc.). Thus, adjectives can be a quite ambiguous and error-generating area as they do not correspond to objects, being states or qualities.

Monosemous lexemes with straightforward definitions in the medical domain may be accompanied by prepositions to form complex structures with an adjective head word. The sample sentences describing the Block's system of classification of caries demonstrate the obligatory attributive adjective use are as follows:

1. Another way of finding mesial and distal cavities at an early stage is transillumination.

2. Caries is located on the proximal surfaces of central and lateral incisors and cuspids.

3. Caries is located in pits and fissures of the occlusal two thirds of posterior teeth or on the lingual_surface of anterior teeth. 
4. Good dental care depends on accurate diagnosis, and accurate diagnosis is based on information.

The following attributive adjectives and their collocations were of highest frequency in the EMP corpus (Dentistry):

\section{A. Key Word list of attributive adjectives (Dentistry):}

maxillary, mandibular, nasal, lacrimal, parietal, temporal, frontal, occipital, ethmoidal, sphenoidal, zygomatic, lingual, lateral, distal, masticatory, salivary, parotid, submandibular, sublingual, labial, external, buccal, mesial, incisal, occlusal, apical, dorsal, internal, alveolar, pterygoid, temporal, squamous, mucous, superior, inferior, coronal, infraorbital, coronoid, condyloid, sagittal, lambdoid, metopic, horizontal, transverse, median, internal, pyramidal, palatine, irreversible, autogenous, advancing, acute, cranial, gingival, partial, etc.

Sentences with impersonal, non-agentive subjects and passive voice constructs were also incorporated in the tests as they constitute part of the obligatory medical English lexico-grammatical instrumentarium. Specific adjectives with an additional semantic component [dimension] and [duration] such as parallel, central, distal, permanent that are obligatory elements of terminological units and collocations are integrated in assessment tasks.

Example: Answer key for a drag and drop task (describing tooth surfaces):

Describing tooth surfaces. Match the adjectives with their definitions

\begin{tabular}{|l|l|}
\hline toward the inside of the cheek & buccal \\
\hline toward the lips & labial \\
\hline toward the tongue & lingual \\
\hline closest to the midline of the face & mesial \\
\hline farthest from the midline of the face & distal \\
\hline cutting edge of the anterior teeth & incisal \\
\hline grinding or chewing surface of all posterior teeth & occlusal \\
\hline relative to the root tip end of tooth & apical \\
\hline facing away from the axis of an organ (back) & dorsal \\
\hline
\end{tabular}

\section{B. Key Word list of predicative adjectives (Dentistry):}

necessary, short, important, high, hard, similar, low, required, useful, complex, suitable, soft, accurate, strong, indirect, mild, rigid, horizontal, parallel, composite, flexible, brittle, durable, perpendicular, deformed, decayed, corrosive, painful, personal, significant, specific, acute, afraid, swollen, adjacent, infective, harmful, 
healthy, untreated, inflamed, irregular, indirect, sterile, susceptible, in/convenient, responsible, etc.

The linking verbs that are found in the medical corpus (be, become, make) are among the most prototypical ones with an impersonal sentence subject. The prepositional phrase and the predicative adjective form a complex predicate. Such core propositions usually require further explanation to specify their meaning. Therefore, structures that contain extensions such as prepositional phrases which modify the predicate and describe in what way or under which condition the proposition is true are extremely frequent in medical English).

Predicative adjective patterns (Wordsmith Tool):

- Evidence on the cariogenicity of these foods is sparse and comes from animal studies. Plaque $\mathrm{pH}$ studies and studies in vitro suggest that maltodextrins and glucose syrups are cariogenic.

- Pain becomes severe, spontaneous and persistent, and is often poorly localized.

- Regional lymphadenopathy is common, and fever may be present.

- Plaque disclosing tablets are available at pharmacies and prescription is not necessary.

- The ADA recommends that consumers replace toothbrushes approximately every 3-4 months or sooner if the bristles become frayed with use.

- Dental caries is becoming less common in developing countries undergoing nutrition changes.

Combining polysemous adjectives with prepositions and adding complements results in specialization of the adjective meaning. The prepositions explicitly introduce a new participant role, thus making the originally rather general meaning of the predicative be + adjective phrase precise and specific. The prepositional noun phrase, marked as [PP], is the second frequent adjective pattern in the corpus. The core predicative adjectives with prepositional complements from the EMP corpus such as [Adj.] $+[$ PP (to/in/for/of/on/as/about/from/over/against/than/as] are included in gap-fill tasks (Table 5).

Prepositional complements (Wordsmith Tool):

- In some European countries (e.g. Norway, Finland, Sweden, Slovenia, Slovakia, Gennany, Croatia or Denmark) orthodontic treatment is available without charge to patients under 18 as benefits for orthodontic treatment are provided under government-run health care systems. 
- In fact, these water schemes were providing 'non-fluoride-safe' drinking water, which is harmful to the residents' health by inducing dental fluorosis and skeletal fluorosis.

- Milk teeth are more susceptible to caries, since the deciduous teeth enamel has a lower mineral content.

- It may also be possible for microorganisms that are present in the environment where the toothbrush is stored to establish themselves on the brush.

- Sealants are especially beneficial for children because their newly erupted, permanent teeth are most susceptible to cavities, however patients of all ages can benefit from dental sealants.

Other lexical patterns that are tested are complementation by a finite clause of the type [it] + [be] + [Adj. (essential, necessary) + [that-CL], and complementation by a non-finite clause of the type [it] + [be] + [Adj. (convenient, easy, available) + [to INF]. Complementation by a finite clause structure relates the adjective to a present or future fact or action that is introduced by a that-clause. Adjectives in this group are either modal or semantically neutral (desirable, suitable, possible), possess no degree of specificity and appear in habitual expressions with anticipatory it. Complementation by a non-finite clause is the most repeated type of complementation introducing processes. The sentences below are examples of extraposition.

Complementation by a clause (Wordsmith Tool)

- It is interesting to note that even the heart cannot survive without blood flowing through the vessels that bring nourishment to its muscular walls.

- In the most severe cases involving bone loss, it is frequently necessary to reshape the rough bone.

- It is recommended that national health authorities and decision-makers formulate country-specific and community-specific goals for reducing the amount of free sugars aiming towards the recommended maximum of no more than $10 \%$ of energy intake.

- We know that the oral cavity is home to hundreds of different types of microorganisms, therefore, it is not surprising that some of these microorganisms are transferred to a toothbrush during use.

Adjectives that are complemented by a [that-clause] relate the subject to a fact. The adjectives in this construction express evaluative attitude towards the content of the clause, but as the sentence has an anticipatory it as subject, the structure is more impersonal. 
Concerning test content, priority is given to the predicative construction [be] + $[\mathrm{Adj}]+\mathrm{C}]$ as it represents the predicator and functions as a main-verb with an extension, introducing various dimensions, describing situation types (dynamic or stative situations).

Further on, terminology and vocabulary retention of nouns as terminological units is incorporated in the design of the following types of tasks: accurate use of keywords and terminological units, identification of collocations (missing parts), use of linking devices and signal words for important information (gap-fill tasks), matching terms with their definitions, word formation tasks, use of reporting verbs, drag and drop activities related to key words (cloze tasks), labelling pictures, etc.

\section{Use of key nouns and verbs}

Example: Answer key for definitions (Dentistry corpus).

Match the definitions with the terms for the structures in the oral cavity.

\begin{tabular}{|l|l|}
\hline - Frenum, frena & $\begin{array}{l}\text { - a connecting fold of membrane serving to support or } \\
\text { restrain any part }\end{array}$ \\
\hline - Palatine raphe & $\begin{array}{l}\text { - the white ridge between in the middle of the hard } \\
\text { palate }\end{array}$ \\
\hline - Papillae & - taste buds \\
\hline - Rugae & - irregular folds or bumps on the surface \\
\hline - Tonsil & $\begin{array}{l}\text { - a mass of lymphatic tissue one on each side of } \\
\text { the oral pharynx }\end{array}$ \\
\hline - Uvula & $\begin{array}{l}\text { - a small pendant fleshy lobe at the back of the soft } \\
\text { palate }\end{array}$ \\
\hline $\begin{array}{l}\text { - Vermillion } \\
\text { border }\end{array}$ & \begin{tabular}{l} 
area where the pink-red lip tissue meets facial skin \\
\hline
\end{tabular} \\
\hline
\end{tabular}

\section{Key Word list of verbs (Dentistry):}

chew, floss, discard, remove, extract, provide, treat, affect, contain, replace, rinse, spit, experience, produce, prevent, protect, erupt, crack, fill, expose, grind, restore, recommend, infect, occur, perform, administer, consume, identify, indicate, suffer, trigger, bleed, reduce, refer, sedate, alleviate, emerge, bond, etc.

Specific verbs in Dentistry (Wordsmith Tool)

- 20. About a third of dentists use nitrous oxide to sedate patients who are anxious. 
- 21. The Vipeholm study was conducted shortly after the Second World War in Sweden between 1945 and 1953.

- 22. More often, one or more of these third molars fails to emerge in proper alignment or fails to fully emerge through the gum line and becomes entrapped or "impacted" between the jawbone and the gum tissue.

- 23. When conservative nonsurgical methods such as positive pressure air machines and dental splint appliances fail to alleviate this problem, surgery can be tried.

- 24. The examination can be supplemented with high-density stone study casts, intra-oral photographs, radiographs and salivary tests.

- 25. A tooth-colored adhesive filling material is bonded to the surface of the tooth, filling the cavity and protecting the tooth surface.

\section{Results}

The most challenging tasks (content-based) during the semester built the dynamic content of the test pools (Fig. 1).

A complete test version (Appendix1) was further adapted and uploaded on the university Blackboard platform for electronic assessment (Fig. 2). Minimum two progress test versions and two final test versions (4/year) were created by the instructor anew to expand the English for Dentistry test bank. All test questions/tasks were archived in a repository bank and could be used in a test by random selection to extend the available samples each time a student clicks on a test to take it. Further on the tasks were designed for two levels of language difficulty (B1 and B2) and the tests were graded (versions A and B) to suit the needs of all students in the mixed level groups in English for medical purposes.

\section{Conclusions}

The corpus-based analysis of teaching resources and textbooks in English for Dentistry highlights a strategic approach to test content and design. The compilation of a corpus of specialized texts guarantees an adequate test content and adherence to a set of recurrent linguistic units and patterns for evaluation of learning outcomes. Emphasis on the characteristic features and the conventions of the academic language for medical purposes (EMP) makes the test relevant. Test banks based on the idea of clusters, collocations and lexical patterns extracted from textbook and teaching materials were organized into folders according to the examination programme (progress and final exams, language practice) and graded for two levels of language proficiency (version A and B for CEFR levels B1 and B2 respectively) with a variation of question types. By using corpus-data for test development, we bring authenticity, reliability, and improved quality of the items 
in the test banks. Another possible advantage of the random compilation of test items from different pools in a bank is the reduced likelihood of cheating.

Fig.1. Final assessment test in English for Dentistry - language level B2: Listening task on Blackboard platform

\section{QUESTION 2}

Listening task 2: Listen about wisdom teeth extraction and choose the correct answer. Wisdom Teeth Removal

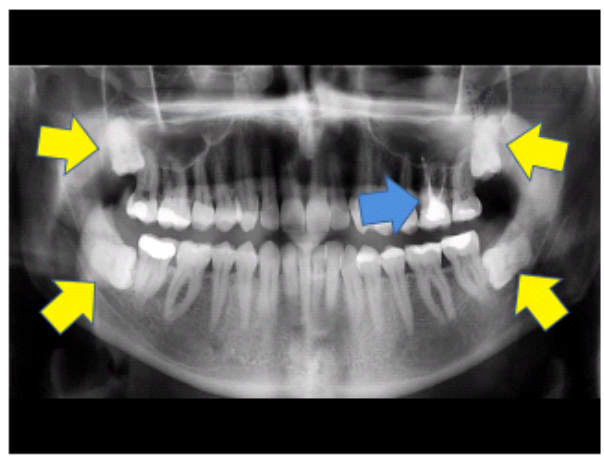

1. Which procedure is required prior to taking the decision for wisdom teeth extraction?
a. X-ray
b. cyst removal
c. none of the above

2. What is not a consequence of impacted wisdom teeth?
a. decay
b. cyst
c. eruption

3. When is wisdom teeth removal recommended? 
Fig. 2: Creating pools, Blackboard platform.

\title{
Pools
}

Pools are sets of questions that you can use in multiple tests or surveys. I

\section{Build Pool Import Pool}

\author{
Name \\ Final Test_Semester II_Listening 2 (T/F с падащுо меню) \\ Final Test_Semester II _Listening 1 (multiple choice) с падащо меню \\ Final Test_Semester II_Listening 1 (урна в цалост) \\ Final Test_Semester II_Listening 2 (урна в цялост) \\ Final Test_Semester II_Reading 1 (fill in the words) \\ Final Test_Semester II_Reading 2 (T/F) \\ Final Test_Semester II_Reading 3 (fill in sentences)
}

\section{Fig. 3. E-assessment in English for Dentistry, MU-Varna}

\section{Tests}

Tests are a means of assessing student performance. You have the option to build a test or import a test. More Help

\section{Build Test Import Test}

\section{Name $\triangle$}

Final Test_2019

Final Test_Semester II_2019 C

Final Test_Semester II_testing

Progress Test Semester I - Retake 2017

Progress Test Semester | 2017

Progress Test Semester I, 2018 (A)

Progress Test Semester I, 2018 (B)

Retake Final Test_2019 C

Sample Test Semester I 2017

\section{Deployed}

Final Test_Semester II

Final Test_Semester II

No

Progress test

Progress test

Progress test

Progress test

Final Test_Semester II

Sample Tests
Date Last Edited

April 25, 2019 6:26 PM

May 9, 2019 3:51 PM

March 21, 2017 3:18 PM

December 18, 2017 4:22 PM

December 18, 2017 8:55 AM

November 28, 2018 1:22 PM

December 6, 2018 9:41 AM

May 9, 2019 9:28 AM

October 16, 2018 1:27 PM 


\section{References:}

Benson, M., Benson, E., \& Ilson, R. (2010). The BBI Combinatory Dictionary. John Benjamins Publishing Company.

Collins Online Dictionary < https://www.collinsdictionary.com/>

Cruse, D. (2011). Meaning in Language: an introduction to semantics and pragmatics. Oxford: Oxford University Press.

Erlangen Valency Patternbank <http://www.patternbank.uni-erlangen.de/cgibin/patternbank.cgi

Doykova, I. (2019). Exploring Student Expectations and Perceptions in English for Medical Purposes (EMP). In Conference Proceedings (pp. 89-94).

Guiding Principles for Quality Textbooks $<$ https://www.edb.gov.hk/en/curriculum-development/resource-

support/textbook-info/GuidingPrinciples/index.html

Herbst, T., \& Schüller, S. (2008). Introduction to Syntactic Analysis: A Valency Approach. Narr Studienbücher Verlag.

Huddleston, R., \& Pullum, G. (2002). The Cambridge Grammar of the English Language. Cambridge University Press.

Lewis, M. (ed.). (2000). Teaching Collocation: Further Developments in the Lexical Approach, London: Thomson Heinle LTP.

Fan, M. (2009). An exploratory study of collocational use by ESL students-A taskbased approach. System, 37(1), 110-123.

Mishan, F. (2005). Designing Authenticity into Language Learning Materials. Intellect Books.

Nesselhauf, N. (2005). Collocations in a Learner Corpus. Amsterdam: John Benjamins Publishing Company.

Rachovska, Y., Angelova, V., \& Trendafiliva, Sv. (2010). English for Dental Medicine.

Steno Publishing House.

Sketch Engine <https://www.sketchengine.eu/

Schmitt, N., \& McCarthy, M. (1997). Vocabulary: Description, acquisition and pedagogy. Cambridge university press.

Scott, M. (2012). Lexical Analysis Software WordSmith Tools version 6.

The British National Corpus <http://corpus.byu.edu/bnc>

Vaughan, E., \& Clancy, B. (2013). Small corpora and pragmatics. In Yearbook of Corpus Linguistics and Pragmatics 2013 (pp. 53-73). Springer, Dordrecht.

\section{Contact}

\section{Ilina Doykova}

Medical University 'Prof. d-r Paraskev Stoyanov' in Varna, Bulgaria

55, Marin Drinov str.

9002 Varna, Bulgaria

ilina.doykova@mu-varna.bg 


\section{APPENDIX}

\section{Listening comprehension.}

\section{Listen to the text on New products for tooth decay management and choose the correct answer:}

1. What is the idea of minimally invasive (MI) dentistry?
A) to assist the clinitians in the treatment of various diseases
B) to treat the causes of oral diseases
C) to focus on new products in dentistry
D) to promote plaque removal

2. What is demineralization?

A) less amount of calcium and phosphate in dental hard tissues

B) the presence of calcium and phosphate in dental hard tissues

C) prevention of tooth decay

D) drilling and filling cavities

3. How does the Tooth Mousse work in the oral cavity?
A) It eliminates bacteria in the mouth
B) It restores the mineral balance within the dental hard tissues
C) It treats sores in the oral cavity
D) It contributes to an improved flow of saliva in the mouth

4. List some positive effects of Oral B Pro-Expert:
A) It facilitates tooth desensitising
B) It contributes to significantly minimize the presence of plaque
C) It helps to preserve the balance in the oral cavity
D) It encourages remineralization and improves the quality of the enamel crystals

5. The presence of fluoride in the plaque and saliva stimulates
A) tooth sensitivity
B) fluoride formulation
C) the antibacterial and anti-inflammatory effect
D) tooth whitening 
6. Which compound is best suited for the demands of MI technique?
A) Biodentine
B) Oral B Pro-Expert
C) GC Tooth Mousse
D) amalgam fillings

7. When did Biodentine first appear on the market?
A) it is still not available for commercial use
B) five years ago
C) it is a promising brand new product
D) in 2010

8. What dosage forms is Biodentine available in?
A) as a syrup
B) in capsules
C) in powder and liquid form
D) as lozenges

9. How do amalgam and composite-based silver fillings work?
A) They preserve the pulp vitality
B) They are used to replace the affected tooth tissue
C) They allow the healing of the tooth
D) The promote generation of new dentine

Total points: 18

\section{Reading comprehension}

Read the text and mark the statements below as True or False:

\section{Pros and Cons of Taking Fluoride supplements}

Some people think taking fluoride dietary supplements available in the form of tablets might help to prevent cavities and other oral conditions. However, these supplements, unlike other similar products, are available only by a doctor's prescription and may not be safe for everyone. Moreover, they should be used according to the suggested dosage, otherwise a condition known as fluorosis may 
occur which is characterized by yellow or brown discoloration patches on permanent teeth due to too much fluoride intake.

Fluorosis can be mild to moderate and even severe with black patches on teeth. Dentists are now spreading awareness to decrease the risks of this treatment to prevent tooth decay. American Dental Association or ADA prescribes fluoride supplements for only those children who live in non-fluoridated areas. For these children, it is recommended that they use fluoride supplements until they reach the age of 16. It has been found that fluoride tablets cause dental fluorosis in $64 \%$ of the children. Moreover, if taken in larger amounts these tablets may even cause death. Instead of prescribing fluoride supplements to children, it is more appropriate to spread awareness about the benefits of breast milk. If infants are given adequate milk in their early months, chances of tooth decay decrease automatically and there is no need for additional dietary supplements or even fluoridated water.

At the same time, fluoride has been found to be useful for the teeth because it slows down the rate at which bacteria in the mouth produce acids and makes a tooth more resistant to the formation of cavities. However, dentists and common people are learning more and more about the dangers of fluoride the most prevalent of which is fluorosis. It is advisable to use fluoride only as a topical treatment, by using gels, toothpastes, or mouth rinses. Toothpastes only contain about $0.1 \%$ fluoride in the form of a compound.

Potential sources of fluoride is the use of too much fluoridated toothpaste at an early age, the inappropriate use of fluoride supplements, and some hidden sources of fluoride in the child's diet.

Two and three-year olds may not be able to expectorate (spit out) fluoridecontaining toothpaste when brushing thus swollowing an excessive amount of fluoride during tooth brushing. Certain foods contain high levels of fluoride, especially powdered infant formula, infant dry cereals, creamed spinach and infant chicken products. Some beverages also contain high levels of fluoride, especially decaffeinated teas, white grape juices and juice drinks manufactured in fluoridated cities. Excess consumption of fluoride through fluoridated water or dietary supplements may cause dental as well as skeletal fluorosis, which is less common.

1. Consuming fluoride supplements exposes children and adults to many adverse health effects including kidney damage and bone fracture. True/False

2. Dentists recommend fluoride therapy for a number of reasons, including reduction of tooth sensitivity, protection of root surface, prevention of tooth decay, and treatment of white spots.

3. Fluoride drops and tablets, as well as fluoride fortified vitamins should not be given to infants younger than six months of age. 
4. The dangers of fluoride therapy for the prevention of tooth decay are more than its benefits and therefore fluoride therapy is not a recommended treatment.

5. Fluoride supplements should only be given to children after all sources of ingested fluoride have been taken into account.

6. Too little or too much fluoride can be harmful to the teeth.

7. Swollowing toothpaste during the period of permanent tooth development is the risk factor in the development of fluorosis.

8. There is no evidence available to support the theory that fluoride tablets prevent tooth decay in children.

Total points: 16

\section{Use of English}

halitosis, further, reabsorbed, concluded, coatings, although, promotes, buds, crevices, thorough, rough, bristles, forward, bent, build-up

\section{Tongue Cleaning Tools}

You have three choices for cleaning your tongue. You can use a tongue scraper which is a long, thin, flat piece of metal that is $\mathbf{1}$. in a "U" shape. Today, you can buy scrapers made from plastic or metal. Simply place the edge of the tool on the back of your tongue, and gently pull the scraper 2 .

Some people use a regular toothbrush to clean the tongue. The disadvantage of using a regular toothbrush on the tongue is that the $\mathbf{3}$. are designed to clean the smooth surfaces of your teeth. Your tongue is a 4. surface with many tiny crevices, and regular toothbrush bristles may not do a $\mathbf{5}$. cleaning job. A toothbrush reduces the production of volatile sulfur compounds by 45 percent while a tongue scraper reduces production by $75 \%$.

Your third choice for tongue cleaning is a tongue brush with bristles designed to clean out the 6 . of the tongue. This relatively recent invention performs with about the same effectiveness as a traditional tongue scraper.

Dental research has 7 . that a tongue scraper is more effective at removing toxins and bacteria from the tongue than a toothbrush. 8. brushing and flossing will loosen and move debris around, they do not actually remove the bacteria. Almost half of our oral bacteria live on and in the deep crevices of our tongue; the scraping action of a tongue scraper collects these toxic tongue 9. (which can range in color from clear, white, yellow, or green) and removes them from the body. If you don't take steps to remove toxic mucus on the tongue, your taste $\mathbf{1 0}$. can become blocked. Removing 11.

from the surface of your tongue will open up its pores and better expose your taste buds. 12. on, the tongue is part of the first line of defense in your immune system. Scraping your tongue prevents toxins from being 13. into your 
body and boosts overall immune function. This oral hygiene practice 14 . general tooth and gum health and removes bacteria and toxins responsible for periodontal problems, plaque build-up, tooth decay, loss of teeth, gum infections, gum recession, and $\mathbf{1 5 .}$

Total points: 30

\section{Read the following definitions and identify the terms they describe:}

1. one of the 8 permanent grinding teeth, 2 in the upper and 2 in the lower jaw, located between the canines and the molars

2. stoppage, preclusion

3. the small piece of soft tissue at the back of the soft palate

4. a tooth that is chisel-shaped and used for cutting

5. the bony part of the roof of the mouth

6. the emergence of a tooth through the gums

7. a sharp and pointed tooth

8. the tissue covering the jaws on the inside of the mouth

9. pain that comes and goes rhythmically

10. mobile muscular tissue in the oral cavity

11. juice produced by the pancreas

12. bowel

13. waste products of the process of digestion

14. nourishment

15. clotting

16. milk teeth

Total points: 16

\section{Audioscript:}

\section{Tooth decay management}

Evidence-based dentistry has caused a shift from the "drill-and-fill" days to practicing minimally invasive (MI) dentistry. In the past, management of tooth decay involved either removal of the decay, by drilling and then filling the resultant cavity, or by extraction of the decayed tooth. The new MI approach accepts that the model of dental decay can be visualized as a balance between the pathological factors promoting (2) loss of calcium and phosphate from the dental hard tissues (demineralisation) and the protective factors promoting the process whereby calcium and phosphate enter the dental hard tissues (remineralisaton). The concept of MI dentistry focuses on (1) treating the causes of oral diseases instead of addressing their symptoms. The following new products are useful tools in the armoury to assist the clinicians advocating minimally invasive dentistry. 
The GC Tooth Mousse is a water-based, sugar-free dental topical cream containing caesin phosphopeptide (amorphous calcium phosphate). This complex is an ideal deliverer of calcium and phosphsate ions to the oral cavity. When applied to the tooth surface, the mousse (3) restores the mineral balance within the dental hard tissues contributing to the reversal of non-cavitated tooth decay lesions and neutralising acid from bacteria found within plaque.

Fluoride-containing toothpastes like Oral B Pro-Expert interfere with the process of tooth decay by a number of ways. It is accepted that the presence of fluoride in the plaque and saliva (4) encourages remineralisation and ensures that the enamel crystals are of improved quality. Unlike traditional sodium fluoride formulation toothpastes, this low-water formulation has a proven effective (5) antibacterial, anti-inflammatory, antiplaque, anti-halitosis, tooth-desensitising and whitening effect. Unfortunately, not all decay carious lesions are treatable with topical remineralising products. Laboratory and in vivo studies have shown this technique to be a promising microinvasive approach to preserve demineralised enamel.

In the advanced stages of tooth decay, intervention necessitates drilling of a tooth to remove the affected hard dental tissues. However, new materials are emerging on the dental market catering for the demands of MI technique. The first of such materials is (6) Biodentine. Biodentine is the first all-in-one bioactive and biocompatible dentine substitute, based on a unique active biosilicate technology. Released in (7) 2010 after undergoing research in 300 patients over five years, Biodentine is seen as the future for tooth restorative materials. Biodentine is an inorganic, non-metallic compound presented in (8) a capsulated powder and liquid form. The powder phase consists of tricalcium silicate, calcium carbonate and zirconium dioxide and the liquid phase combines calcium chloride, water and a water reducing agent. Unlike amalgam or composite-based silver fillings, which (9) replace affected tooth tissue and may work to allow healing of the tooth pulp, Biodentine's bioactivity helps to preserve pulp vitality in deep cavities by promoting generation of new dentine. Its use in a range of endodontic scenarios for the treatment of dental nerve conditions is promising. /475 words/ 